\title{
THE PROTECTIVE ROLE OF CYTOTOXIC T CELLS AND INTERFERON AGAINST CORONAVIRUS INVASION OF THE BRAIN
}

\author{
Ehud Lavi and Qian Wang \\ Division of Neuropathology \\ Department of Pathology and Laboratory Medicine \\ University of Pennsylvania School of Medicine \\ Philadelphia, Pennsylvania 19104-6079
}

\begin{abstract}
MHV-A59 causes focal acute encephalitis, acute hepatitis, and chronic demyelination while MHV-2 causes acute hepatitis and no brain involvement. The difference in organ tropism between these two closely related MHVs is not related to the ability of these viruses to grow in brain cells since both viruses grow equally well in primary glial cell cultures derived from neonatal mouse brains. We postulated therefore that the ability of the virus to stimulate certain host immunological factors may be important for protection of the brain against invasion and replication of the virus. In this study we performed preliminary experiments to investigate the potential role of two host factors in protection of the brain against MHV invasion: cytotoxic T cells and interferon.

Four week old $\beta 2 \mathrm{M}^{(-/)}$mice, lacking $\beta 2$ microglobulin, MHC class I expression and functional cytotoxic CD8 $+\mathrm{T}$ cells were inoculated intracerebrally (IC) with MHV-2 and analyzed at various intervals post infection for histopathology and viral titers in organs. Histology revealed both acute hepatitis and acute encephalitis. Acute encephalitis was observed in periventricular areas. Mononuclear lymphocytic infiltration involved the choroid plexus, the ependyma and in the surrounding brain parenchyma. There was no involvement of other areas of the brain including areas that are typically involved in A59 infection of $\mathrm{C} 57 \mathrm{Bl} / 6$ mice. By contrast, $\mathrm{C} 57 \mathrm{Bl} / 6$ mice infected with MHV-2 showed no involvement of the brain parenchyma and only slight inflammation of the choroid plexus was present. High titers of infectious virus was detected by plaque assay in both brains and livers of $\beta 2 \mathrm{M}^{(-/)}$mice infected with $\mathrm{MHV}-2$ in contrast to only liver titers in $\mathrm{C} 57 \mathrm{Bl} / 6$ mice infected with a similar dose of MHV-2.

Polyclonal rabbit-anti mouse IFN $\alpha / \beta$ or anti IFN $\beta$ (Lee Biomolecular Research $\mathrm{LAb}$.) was given to groups of 4-week-old $\mathrm{C} 57 \mathrm{Bl} / 6$ mice at a dose of $10,000 \mathrm{U}$ per one I.P. treatment, 24 hours prior to I.C. inoculation of $1 \mathrm{LD} 50$ of MHV-2 or MHV-A59. At various intervals post inoculation virus titers from brains and livers were determined by plaque assay, and the histopathology of all the internal organs was analyzed by H\&E staining. Treatment
\end{abstract}


with preimmune serum from the same rabbit was used as control with no effect on disease outcome in either one of the viruses. While IFN antibodies had little or no effect on the outcome of disease in MHV-A59 infection, mice treated with either anti IFN $\alpha / \beta$ or anti IFN $\beta$ had high titers of virus recovered from the brain and histopathological evidence of acute meningoencephalitis. Thus cytotoxic $\mathrm{T}$ cells and interferon may have a protective role against brain invasion of the virus in MHV-2 infection in mice.

\section{INTRODUCTION}

The ability of viruses to replicate and invade the brain is a function of the availability of cellular receptors for the virus, the ability of the virus to gain access into neural tissues (neuroinvasiveness) and a variety of host immunological barriers. Some coronaviruses contain properties of neurovirulence and neuroinvasiveness while other closely related strains are lacking these properties. For example MHV-A59 causes focal acute encephalitis, acute hepatitis, and chronic demyelination while MHV-2 causes acute hepatitis and no brain involvement. The difference in neurotropism in these viruses provides an excellent tool for the study of molecular control of neurotropism and protection of the brain from viruses. The difference in organ tropism between these two closely related MHVs is not related to the ability of these viruses to grow in brain cells since both viruses grow equally well in primary glial cell cultures derived from neonatal mouse brains. We postulated therefore that the ability of MHV to stimulate certain host immunological factors may be important for protection of the brain against invasion and replication of the virus. In this study we performed preliminary experiments to investigate the potential role of two host factors: cytotoxic T cells and interferon.

\section{Cytotoxic T Cells}

Cytotoxic T cells, $\mathrm{CD}^{+}$, are important for clearance of MHV infection ${ }^{1}$. Our previous experiments with acute MHV-A59 infection in $\beta 2 \mathrm{M}^{(-/)}$mice revealed that inflammatory response in the brain is delayed in the absence of functional CD8+ cells, suggesting that cytotoxic T cells are an early inflammatory factor during MHV infection of the brain. Their protective action may be either by direct cytotoxic phenomenon or via the secretion of antiviral cytokines such as interferon.

\section{Interferon}

Using a comparative analysis between the neurotropic MHV (A59), a non-neurotropic MHV (MHV-2) and a set of recombinants between the two viruses, we are studying the molecular control of viral neurotropism. In our preliminary correlation between the phenotypic analysis and the nucleic acid sequence analysis of these viruses, it appeared that the $\mathrm{M}$ gene may be a determinant of acute encephalitis. A region at the 5' end of the $\mathrm{M}$ gene has been shown to control the ability of another coronavirus (TGEV) to induce interferon response ${ }^{2}$. Some coronaviruses such as TGEV are strong interferon inducers ${ }^{3}$ while others such as MHV-JHM are not. Thus we wanted to test whether treatment with anti-interferon antibodies have an effect on the outcome of disease and organ tropism in MHV infection.

\section{EXPERIMENTAL DESIGN AND METHODS}

To investigate the role of $\mathrm{CD}^{+}$cells in acute MHV-A59 infection we studied infection of $\beta 2$ microglobulin negative transgenic mice. The expression of $\beta 2$ microglobulin 
on the surface of cells is closely associated with the expression of the MHC class I protein, thus disruption of $\beta 2$ microglobulin expression interferes with the normal expression of MHC class I. The cytotoxic T cell reaction is highly dependent upon recognition of antigens in the context of MHC class I. Furthermore, the development of $\mathrm{CD}^{+}$cells is completely dependent upon MHC class I expression. The $\beta 2 \mathrm{M}^{(-)}$knockout mice are therefore devoid of $\mathrm{MHC}$ class I expression and therefore lacking functional $\mathrm{CD}^{+}$cells.

Four week old $\beta 2 \mathrm{M}^{(--)}$mice, originally obtained from Dr. Koller ${ }^{4}$, were inbred at the University of Pennsylvania, and were inoculated intracerebrally (IC) with MHV-2. Groups of 3-5 mice per time point (days 1,3,5,7,9, 11, days post inoculation) were sacrificed by anesthetic (methoxyflurane) overdose and perfused intracardially with PBS and $10 \%$ phosphate buffered formalin. Organs were removed and fixed in $10 \%$ phosphate buffered formalin. Tissues were embedded in paraffin, sectioned and stained with H\&E for light microscopy 5 .

Polyclonal rabbit-anti mouse IFN $\alpha / \beta$ or anti IFN $\beta$ (Lee Biomolecular Research $\mathrm{LAb}$.) was given to groups of 4-week-old $\mathrm{C} 57 \mathrm{Bl} / 6$ mice at a dose of $10,000 \mathrm{U}$ per one I.P. treatment, 24 hours prior to I.C. inoculation of $1 \mathrm{LD} 50$ of MHV-2 or MHV-A59 ${ }^{6}$. At various intervals after inoculation virus titers from brains and livers were determined by plaque assay, and the histopathology of all the internal organs was analyzed by H\&E staining.

\section{RESULTS}

\section{Infection of $\beta 2 \mathrm{M}^{(-/)}$Knockout Mice with MHV-2}

Light microscopy histopathologic examination of $\mathrm{H} \& \mathrm{E}$ stained sections of $\beta 2 \mathrm{M}^{(-/)}$ mice infected I.C. with $1 \mathrm{LD}_{50}$ dose of $\mathrm{MHV}-2$ revealed involvement of both brain and liver. During the acute stage of disease both acute hepatitis and acute encephalitis developed. Acute encephalitis was centered around the periventricular areas around the lateral ventricles, third and fourth ventricles. In all of these locations mononuclear lymphocytic infiltration involved the choroid plexus, the ependyma and slightly infiltrating in the surrounding parenchyma in the form of perivascular inflammation of the Virchow-Robin spaces and pericapillary. There was no involvement of other areas of the brain including areas that are typically involved in
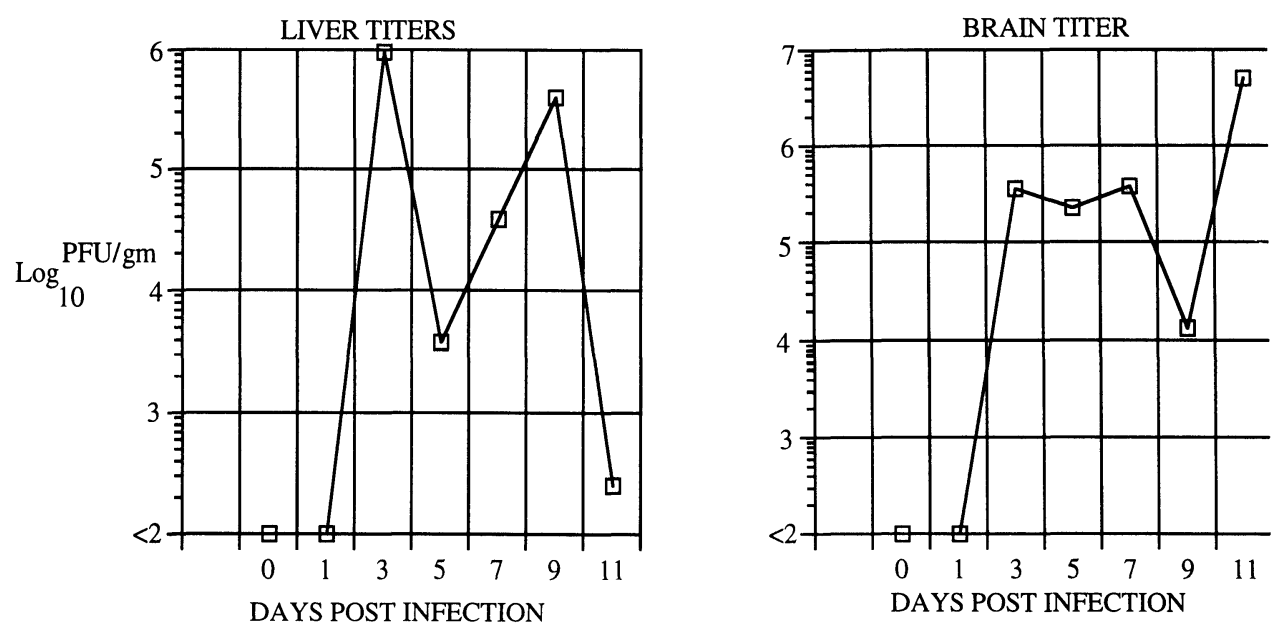

Figure 1. Organ titers following infection of $\beta 2 \mathrm{M}^{(-/)}$mice with $1 \mathrm{LD}_{50}$ dose of $\mathrm{MHV}-2$. 
Table 1. Histopathological changes in C57Bl/6 mice receiving MHV-2 following treatment with rabbit polyclonal antibodies against interferon alpha/beta. Alternatively mice were inoculated intracerebrally with MHV-2 following treatment with preimmune rabbit serum

\begin{tabular}{lccccccc}
\hline & & \multicolumn{7}{c}{ PID } \\
\cline { 2 - 8 } & & 1 & 3 & 5 & 7 & 9 & 11 \\
\hline MHV-2 & H & - & + & +++ & +++ & + & \\
pre-immune sera & T & & & + & +++ & - & \\
& M & - & - & - & - & - & \\
& E & - & - & - & - & - & \\
MHV-2 & C & - & - & + & + & + & \\
IFN $\delta / \beta$ abs & H & - & + & +++ & ++ & ++ & + \\
& T & & & - & & +++ & - \\
& M & + & + & + & - & - & - \\
& E & - & - & ++ & + & + & - \\
& C & - & - & + & + & + & + \\
\hline
\end{tabular}

Key: PID - post inoculation day; $\mathrm{H}$ - hepatitis; $\mathrm{T}$ - thymic cortical depletion of lymphocytes; M - meningitis; E - encephalitis; C- choroiditis (choroid plexus inflammation).

A59 infection of $\mathrm{C} 57 \mathrm{Bl} / 6$ mice. By contrast, C57Bl/6 mice infected with MHV-2 showed no involvement of the brain parenchyma and only slight inflammation of the choroid plexus was present.

To examine the growth of MHV-2 in the organs of infected mice and to rule out that inflammation of the brain was in this case a reactive non specific process we examined the organ titers at various time points after infection. The average of 2 mice per time point was assessed by plaque assay on L2 monolayers in six well plates inoculated with ten fold dilutions of the samples and is expressed in $\log _{10}$ of $\mathrm{pfu} / \mathrm{gm}$ tissue. High titers of infectious virus observed in both brain and liver in contrast to only liver titers in C57B1/6 mice infected with a similar dose of MHV-2.

\section{Anti-Interferon Treatment}

While IFN antibodies had little or no effect on the outcome of disease in MHV-A59 infection, in mice treated with either anti IFN $\alpha / \beta$ or anti IFN $\beta$ prior to infection with MHV-2, high titers of virus was recovered from the brain and histopathological evidence of acute meningitis and encephalitis was seen in H\&E stained sections of brains (Table 1). Treatment with preimmune serum from the same rabbit was used as control with no significant effect on disease outcome in either MHV-2 or A59.

\section{DISCUSSION}

MHV-2 and A59 are closely related strains of MHV and share a high degree of molecular homology. However, the two strains differ substantially in pathogenesis and biologic properties. The main difference is in neurotropism. While A59 is neurotropic and produces acute encephalitis and chronic demyelination, MHV-2 is restricted in its ability to 
invade the brain even when the virus is introduced directly into the brain by I.C. inoculation. The protection of the brain in MHV-2 is not due to lack of receptors on brain cells for the virus since glial cells in culture can be infected equally with both viruses. Thus we considered the possibility that MHV-2 is capable of induction of an immunological response that protects the brain from further invasion of the virus. A59 may be defective in its ability to produce that immunological response. To begin to analyze the possible role of immune cells and cytokines in this phenomenon we asked whether mice lacking CD8+ cells or treated with anti interferon alpha/beta express more susceptibility to MHV-2 infection. In both cases we found that the brain was more susceptible to infection with $\mathrm{MHV}-2$ although $\beta 2 \mathrm{M}^{(-/)}$mice did not express the same distribution of viral infection as $\mathrm{C} 57 \mathrm{Bl} / 6$ mice infected with A59. Thus other factors of the immune system may play a additional role in protecting the brain against MHV-2 invasion.

\section{ACKNOWLEDGMENTS}

This study was supported in part by a grant from the University of Pennsylvania Research Foundation, and by National Multiple Sclerosis Society grants PP-0284 and RG-2615A1/2 (EL).

\section{REFERENCES}

1 Sussman MA, Shubın RA, Kyuwa S, Stohlman SA Cell mediated clearance of mouse hepatitıs virus strain JHM from the central nervous system J Virol 1989,63·3051-3056

2 Laude H, Gelfi J, Lavenant L, Charley B Single amıno acid changes in the viral glycoprotein M affect induction of interferon by the coronavirus transmissible gastroenteritis virus J Virol 1992,66:743-749

3 Laude H, Rasschaert D, Delmas B, Godet M, Gelfi J, Charley B Molecular biology of transmissible gastroenteritis virus Vet Microbiol 1990,23 147-154

4 Koller BH, Marrack P, Kappler JW, Smithies O Normal development of mice deficient in $\beta 2 \mathrm{M}, \mathrm{MHC}$ class I proteins, and CD8+ T cells Science 1990,248 1227-1230

5 Lavı E, Gılden DH, Hıghkın MK, Weiss SR The organ tropısm of mouse hepatıtıs virus A59 is dependent on dose and route of inoculation Lab Anım Sc1 1986,36 130-135

$6 \mathrm{Su} \mathrm{Y-H,} \mathrm{Oakes} \mathrm{JE,} \mathrm{Lausch} \mathrm{RN} \mathrm{Ocular} \mathrm{avirulence} \mathrm{of} \mathrm{a} \mathrm{herpes} \mathrm{simplex} \mathrm{virus} \mathrm{type} 1$ strain is associated with heightened sensitivity to alpha/beta interferon J Virol 1990,64 2187-2192 